\title{
BENEFÍCIOS FUNCIONAIS DA FISIOTERAPIA PARA PACIENTES EM HEMODIÁLISE
}

\author{
Roberta Maria Góes de Souza ${ }^{a}$ \\ Lorena Barreto Arruda Guedes ${ }^{b}$
}

\begin{abstract}
Resumo
Introdução: A população de pacientes com doença renal crônica (DRC) vem aumentando a cada ano, assim como os pacientes em terapia renal substitutiva: hemodiálise (HD). Programas de treinamento físico aeróbico e/ou resistidos de moderada ou baixa intensidade no período interdialítico e durante a hemodiálise têm sido utilizados. Objetivo: Identificar os benefícios dos diferentes tipos de treino de exercício: aeróbico, resistido e a combinação de ambos, nos pacientes com DRC em tratamento dialítico. Metodologia: Foram realizadas buscas de artigos originais e de revisão de literatura sobre o tema, em banco de dados como Scielo, Bireme, Lilacs, Pubmed e Medline, publicados entre 2004 a 2013. Resultados e Discussão: Foram analisados um total de treze artigos que relataram a prática de exercícios físicos em pacientes portadores de DRC, e seus benefícios. Conclusão: Observaram-se nesses estudos, benefícios associados aos exercícios fisioterapêuticos em portadores de DRC em uso de HD. Nesse sentido, a fisioterapia contribui de forma significativa na prevenção, no retardo da evolução e na minimização de complicações apresentadas pelo paciente renal.
\end{abstract}

Palavras-chave: Doença renal crônica; Fisioterapia; Exercícios físicos; Hemodiálise.

\section{FUNCTIONAL BENEFITS OF PHYSICAL THERAPY FOR PATIENTS IN HEMODIALYSIS}

\begin{abstract}
Introduction: The population of patients with chronic kidney disease (CKD) keeps rising every year, as well as the number of patients in renal replacement therapy: hemodialysis (HD). Cardio and resistance physical activity programs (moderate to low intensity) have been utilized in the interdialytic period

a. Bacharel em Fisioterapia, graduada pelo Centro Universitário Estácio da Bahia, pós-graduanda em Fisioterapia Hospitalar na Escola Bahiana de Medicina e Saúde Pública (EBMSP). beta.2@hotmail.com

b. Fisioterapeuta, docente da Pós-Graduação em Fisioterapia Hospitalar EBMSP
\end{abstract}


and also during hemodialysis. Objective: Identify the benefits of those exercises (cardio, resistance exercises and also a combination of both) in the patients with CKD in dialysis. Methods: Pre-reviewed of original articles and literature reviews about the subject, from databases such as Scielo, Bireme, Lilacs, Pubmed and Medline. Publishing dates of those articles and reviews date from 2004 to 2013 . Results and Discussion: A total of thirteen articles were analyzed, all of which discuss the practice and benefits of physical exercises in CKD patients. Conclusion: Many benefits were linked to the therapeutical physical exercises in CKD patients in hemodialysis. In that sense, physical therapy contributes significantly in the prevention, in slowing the progress and overall improvement of complications commonly associated with CKD patients.

Kepwords: Chronic kidney disease; Physical therapy; Physical exercises; Hemodialysis.

\section{INTRODUÇÃO}

A doença renal crônica (DRC) é definida segundo a Sociedade Brasileira de Nefrologia, como uma perda lenta, progressiva e irreversível das funções renais'. Uma condição na qual os rins não apresentam mais funcionalidade por resultado da destruição dos néfrons, resultando na incapacidade do organismo em manter o equilíbrio metabólico e hidroeletrolítico renal ${ }^{2}$. Entre as causas da DRC podemos destacar: diabetes mellitus, hipertensão arterial, glomerulonefrites, doenças císticas dos rins, nefrite intersticial, nefropatia obstrutiva, doenças vasculares do colágeno, malignidades, entre outras. ${ }^{(3)}$

O tratamento de escolha substitutivo da função renal mais utilizado é a hemodiálise (HD), procedimento no qual o sangue é removido do corpo e circulado através de um aparelho externo denominado dialisador. Exige o acesso repetido à corrente sanguínea, geralmente realizado através de uma fístula arteriovenosa que é criada cirurgicamente. ${ }^{(2)}$

Segundo o Censo de Diálise de 2012, o Brasil possui 651 unidades para procedimento dialítico. Nesses serviços $84 \%$ dos pacientes realizaram o tratamento pelo Sistema Único de Saúde (SUS). No perfil dos pacientes atendidos $57,7 \%$ eram do sexo masculino, e estavam na faixa etária entre 19 a 64 anos $(63,6 \%) \cdot{ }^{(4)}$
Pacientes em HD são menos ativos, apresentam baixa tolerância ao exercício e alto descondicionamento físico, provavelmente relacionados à atrofia muscular, anemia, miopatia e neuropatia urêmica, disfunção autonômica, diminuição da flexibilidade, redução da força muscular, má nutrição e comorbidades associadas. ${ }^{(5)}$

Contudo, a literatura demonstra que diferentes tipos de treinos têm sido realizados com pacientes em HD: aeróbico, de resistência e a combinação de ambos. ${ }^{(2)}$

O exercício físico durante o procedimento dialítico pode ter efeitos cardiovasculares positivos, e prevenir ou reverter perda de massa muscular em pacientes em HD crônica. ${ }^{(6)}$ Assim como promover melhora da capacidade funcional (CF), da função cardíaca, da força e resistência muscular. ${ }^{(7)}$

$A$ inserção do fisioterapeuta em uma equipe interdisciplinar que cuida dos pacientes com DRC em HD mostra-se importante, pois vai proporcionar benefícios na condição física, e consequentemente uma melhora na qualidade de vida. (3)

O presente estudo teve como objetivo, identificar os benefícios dos diferentes tipos de treino de exercício: aeróbico, resistido e a combinação de ambos, nos pacientes com DRC em HD. Contribuindo 
para o conhecimento do tratamento fisioterapêutico, nesse perfil de pacientes.

\section{METODOLOGIA}

A pesquisa constou de uma revisão de literatura sobre o tema, em banco de dados como Scielo, Bireme, Lilacs, Pubmed e Medline. Os critérios de inclusão para o trabalho, foram artigos que abordassem os benefícios dos tipos de treino de exercício físico nos pacientes com DRC em HD, publicados entre 2004 a 2013. Foram excluídos da pesquisa, artigos que os pacientes não estivessem em tratamento dialítico para a DRC, e que tivessem uma amostra menor do que dez pacientes. As seguintes palavras-chave, e suas correlatas em inglês e espanhol, foram usadas: doença renal crônica, fisioterapia, exercícios físicos, hemodiálise.

\section{RESULTADOS E DISCUSSÃO}

Foram encontrados no total 44 artigos, os quais 31 atendiam aos critérios de exclusão previamente estabelecidos. Consentindo aos critérios de inclusão, foram selecionados 13 artigos, sendo nove artigos originais (Anexo: tabela 1) e quatro artigos de revisão bibliográfica, abordando o tema proposto, e dentro do período descrito.

$\mathrm{Na}$ literatura, é pouco discutida a prática de exercícios físicos em pacientes em HD. Os estudos analisados ${ }^{(2,3,7-13)}$ sobre o tema possuem protocolos variáveis quanto ao tipo do exercício, frequência, duração e intensidade nos programas propostos, fato que dificultou um consenso de qual melhor treino visando os benefícios para os pacientes em HD.

Com ênfase no treino aeróbico, Henrique et al, (9) estudando 14 pacientes durante o processo dialítico propôs um protocolo de exercício, como fase de aquecimento, condicionamento e resfriamento, utilizando um ciclo ergômetro (por 30 minutos) para prática do treino aeróbico, em um período de doze semanas, com três sessões semanais. Ao final do período do protocolo, observaram discreto aumento do Kt/V (índice adimensional, que mede a depuração da ureia, com base na distribuição dessa na água corporal total, considera-se como ideal $\mathrm{Kt} / \mathrm{V} \geq 1,4$ ) acompanhado de redução significante da creatinina, indicando tendência na melhora da qualidade de diálise, um aumento da CF verificada no teste de caminhada de seis minutos (TC6'), além da redução nas pressões arteriais sistólica (PAS), diastólica (PAD) e média (PAM).

Também usando o ciclo ergômetro, com um treino aeróbico de baixa intensidade (40\% da capacidade máxima de exercício), por um tempo maior (três horas), em dois dias com intervalo de uma semana, em dez pacientes estáveis, Giannaki et al(13) corroboram com os achados anteriores quanto as questões relacionadas à redução da creatinina e da taxa de ureia, além da diminuição nos níveis séricos de potássio. Tal efeito da remoção da ureia, creatinina e potássio, melhoraram a eficiência da HD, a redução de sua duração em 30 minutos, e não trouxe efeitos adversos. ${ }^{(14)}$

Diferentemente dos estudos citados à cima, Kosmadakis et al ${ }^{(12)}$ propuseram durante seis meses, um treino de condicionamento aeróbico com a prática de caminhada regular, previamente à HD. $\mathrm{Na}$ amostra de 40 pacientes com DRC em estágios quatro e cinco, acompanhados prospectivamente, 20 pacientes foram alocados no grupo de exercício. Concluíram que houve melhora na tolerância ao exercício, perda de peso, melhora cardiovascular, evitando um aumento medicamentoso para pressão arterial.

Reboredo et al, ${ }^{(8)}$ aplicaram um protocolo de exercícios semelhantes ao de Henrique et al, ${ }^{(9)}$ em uma amostra de 22 pacientes, divididos em grupo controle e grupo exercício, e não encontraram modificação da variabilidade da frequência cardíaca nem melhora da função ventricular esquerda, a partir da avaliação ecocardiográfica e do exame de Holter.

A realização de exercícios aeróbicos em pacientes com DRC em HD, foi capaz de promover redução da $\operatorname{PAS}^{(6,15)}$ de repouso, melhora os valores da pressão inspiratória máxima $\left(\mathrm{Pi}_{\text {máx }}\right)$, pressão 
expiratória máxima $\left(\mathrm{Pe}_{\text {máx }}\right)$ e o consumo máximo de oxigênio $\left(\mathrm{VO}_{2 \text { máx }}\right) \cdot \cdot^{(15)}$

Quando aplicados protocolos com exercícios resistidos e de alongamentos, por um período que variou de três a quatro meses, com duas a três sessões semanais, foram encontrados melhora da CF, aumento da força e resistência muscular em pesquisas ${ }^{(3,11)}$ com pacientes durante a HD.

Rocha et al, (2) avaliaram a capacidade respiratória e a qualidade de vida dos pacientes com DRC, submetidos a um protocolo de exercícios para membros superiores com técnica de facilitação neuromuscular proprioceptiva (FNP) e respiração diafragmática, exercício de fortalecimento para membros inferiores e exercício com bola exercitadora para preensão manual. Encontraram o pico de fluxo expiratório (PFE) com aumento significante, além de melhora clínica, mas sem significância estatística nos oito domínios do "The Short Form Health Survey" (SF-36), exceto em "vitalidade" que diminuiu.

No entanto, Soares et al,(7) utilizando programas de reabilitação com treinamento de exercícios resistido e alongamentos durante a HD, encontraram benefícios na qualidade de vida nos domínios do SF-36: CF, nível de dor, vitalidade e saúde mental. Tais ganhos podem ser justificados, pelo efeito fisiológico do treino resistido no desenvolvimento da tensão normal do músculo, auxiliando o retorno venoso, e assim, atenuando a perda rápida de líquido durante a HD, além de promover a força muscular necessária para a realização de atividades de vida diárias (AVD's). ${ }^{(3)}$

Lopes et al ${ }^{(10)}$ realizaram um estudo com 15 pacientes com DRC, durante um mês. Aplicando um protocolo de exercício físico ativo livre, de componente isotônico de baixa intensidade para membros superiores e inferiores, que constou de: alongamentos, diagonais de Kabat, rotação de tronco (com bastão), agachamento em cadeia cinética fechada, todos realizados em duas séries de dez repetições previamente à $\mathrm{HD}$. Os autores compararam o Kt/V do mês anterior, com os obtidos após a aplicação do programa proposto, e os valores encontrados não foram capazes de demonstrar a eficiência dos exercícios na melhora dialítica. Porém, observaram que o comportamento psicossocial destes pacientes mudou, houve socialização e melhora do quadro depressivo, além de diminuição de queixas como câimbras, náuseas e vômitos.

Apesar da diversidade quanto à forma de aplicação dos programas de exercícios em pacientes com DRC em tratamento com HD, quanto ao tipo, intensidade, frequência e duração, não há consenso de qual traz mais benefícios funcionais.

\section{CONCLUSÃO}

Nos estudos avaliados, foram observados benefícios associados aos exercícios fisioterapêuticos em portadores de DRC em uso de HD, como: melhora da saúde mental, da $C F$, da resistência e força muscular, e eficiência da HD, além da diminuição da pressão arterial de repouso e uso de medicações anti-hipertensivas, dentre outros. Portanto, se faz necessário que novas pesquisas sejam realizadas para traçar um perfil de condutas aplicadas pelos fisioterapeutas, os quais são capazes de contribuir de forma significativa na prevenção, no retardo da evolução e na minimização de complicações apresentadas pelo paciente renal.

\section{REFERÊNCIAS}

1. Sociedade Brasileira de Nefrologia. [Disponível em: http://www.sbn.org.br] Acessado em 20 de agosto de 2013.

2. Rocha, E. R.; Magalhães, S. M.; Lima, V. P. Repercussão de um protocolo fisioterapêutico intradialítico na funcionalidade pulmonar, força de preensão manual e qualidade de vida de pacientes renais crônicos. J. Bras. Nefrol. 2010 out./dez;32(4).

3. Magalhães, H. G. et al. Análise da eficácia do atendimento fisioterapêutico em pacientes com doença renal crônica em hemodiálise. $2^{\circ}$ Congresso Brasileiro de Extensão Universitária; 1215 set. 2004. Belo Horizonte, MG: UFMG; 2004. 
4. Censo de diálise 2O12. [Acesso 22 Ago. 2O13]. Disponível em: http://www.sbn.org.br/pdf/ publico2O12.pdf

5. Bohm, J.; Monteiro, M. B.; Thomé, F. S. Efeitos do exercício aeróbio durante a hemodiálise em pacientes com doença renal crônica: uma revisão da literatura. J. Bras. Nefrol. $2 \mathrm{Ol} 2$ abr./ jun;34(2).

6. Reboredo, M. M. et al. Exercício físico em pacientes dialisados. Rev. Bras. Med. Esporte. 2007 nov./dec;13(6).

7. Soares, K. T. A. et al. Eficácia de um protocolo de exercícios físicos em pacientes com insuficiência renal crônica, durante o tratamento de hemodiálise, avaliada pelo SF36. Fisioter. Mov. 2011 jan./mar;24(1).

8. Reboredo, M. M. et al . Efeito do exercício aeróbico durante as sessões de hemodiálise na variabilidade da frequência cardíaca e na função ventricular esquerda em pacientes com doença renal crônica. J. Bras. Nefrol. 2010 out./ dez;32(4).

9. Henrique, D. M. N. et al. Treinamento aeróbico melhora a capacidade funcional de pacientes em hemodiálise crônica. Arq. Bras. Cardiol. 2010 jun;94(6).

1O. Lopes, F. S. et al. Influência do exercício isotônico pré-dialítico. Arq. Ciênc. Saúde. 2008 out/dez;15(4):170-5.

11. Oliveros, M. S. R. et al. Estudio piloto sobre entrenamiento físico durante hemodiálisis. Rev. méd. Chile. 2011 ago;139(8).

12. Kosmadakis, G. C. et al. Benefits of regular walking exercise in advanced pre-dialysis chronic kidney disease. Nephrology Dialysis Transplantation. 2011;27:997-1004.

13. Giannaki, C. D. et al. The effect of prolonged intradialytic exercise in hemodialysis efficiency indices. Asaio Journal. 2011;57(3):213-18.

14. Moura, R. M. F. et al. Efeitos do exercício físico durante a hemodiálise em indivíduos com insuficiência renal crônica: uma revisão. Fisioterapia e Pesquisa. 2008;15(1):86-91.

15. Najas, C. S. et al. Segurança e eficácia do treinamento físico na insuficiência renal crônica. Rev. Bras. Med. Esporte. 2009 set./out15(5).

\section{ANEXO 1}

\begin{tabular}{|c|c|c|c|c|}
\hline AUTORES & AMOSTRA & PERÍODO & EXERCÍcIOS & RESULTADOS \\
\hline $\begin{array}{l}\text { Henrique et al } \\
(2010)\end{array}$ & 14 & $\begin{array}{l}12 \text { semanas, três } \\
\text { vezes por semana } \\
\text { (durante a HD). }\end{array}$ & $\begin{array}{l}\text { 1) Alongamentos de MMII, } \\
\text { ciclo ergômetro com baixa } \\
\text { rotação e menor carga. } \\
\text { 2) Exercício aeróbico no } \\
\text { ciclo ergômetro por 3O'. 3) } \\
\text { Alongamentos, exercício } \\
\text { aeróbico com carga e } \\
\text { rotação baixa. }\end{array}$ & $\begin{array}{l}\text { Kt/V, da creatinina, } \\
\text { da PAS, PAD e } \\
\text { PAM, e da distância } \\
\text { percorrida no TC6' e } \\
\text { melhora da anemia. }\end{array}$ \\
\hline $\begin{array}{l}\text { Reboredo et al } \\
(2010)\end{array}$ & $\begin{array}{l}\text { 22, grupo } \\
\text { controle } \\
(n=11) \text { e } \\
\text { exercício } \\
(n=11)\end{array}$ & $\begin{array}{l}12 \text { semanas, três } \\
\text { vezes por semana } \\
\text { (durante a HD). }\end{array}$ & $\begin{array}{l}\text { 1) Alongamentos de MMII, } \\
\text { ciclo ergômetro com baixa } \\
\text { rotação e menor carga. } \\
\text { 2) Exercício aeróbico no } \\
\text { ciclo ergômetro por 35'. } \\
\text { 3) Exercício aeróbico com } \\
\text { carga e rotação baixa. }\end{array}$ & $\begin{array}{l}\text { Não modificou a } \\
\text { variabilidade da } \\
\text { FC, nem promoveu } \\
\text { melhora significante } \\
\text { na função ventricular } \\
\text { esquerda. }\end{array}$ \\
\hline
\end{tabular}


(continuação)

\begin{tabular}{|c|c|c|c|c|}
\hline AUTORES & AMOSTRA & PERÍODO & EXERCÍCIOS & RESULTADOS \\
\hline $\begin{array}{l}\text { Kosmadakis } \\
\text { et al (2O11) }\end{array}$ & $\begin{array}{l}\text { 4O, grupo } \\
\text { controle } \\
(n=20) \text { e } \\
\text { exercício } \\
(n=20)\end{array}$ & $\begin{array}{l}\text { Seis meses, cinco } \\
\text { vezes na semana } \\
\text { (previamente à } H D \text { ). }\end{array}$ & Caminhada por 30'. & $\begin{array}{l}\text { Melhora na } \\
\text { tolerância ao } \\
\text { exercício, perda } \\
\text { de peso, e melhora } \\
\text { cardiovascular } \\
\text { com o uso de } \\
\text { medicamentos para } \\
\text { controle da PA. }\end{array}$ \\
\hline $\begin{array}{l}\text { Giannaki et al } \\
(2011)\end{array}$ & 10 & $\begin{array}{l}\text { Duas semanas. } \\
\text { Protocolo exercido } \\
\text { no mesmo dia no } \\
\text { meio da semana, } \\
\text { começava 30' } \\
\text { depois do início da } \\
\text { diálise, e terminava } \\
\text { 30' antes do } \\
\text { término da diálise. }\end{array}$ & $\begin{array}{l}\text { Exercício no ciclo ergômetro } \\
\text { durante três horas com } 40 \% \\
\text { da capacidade máxima. }\end{array}$ & $\begin{array}{l}\text { da taxa de } \\
\text { creatinina, ureia e } \\
\text { dos níveis séricos de } \\
\text { potássio. }\end{array}$ \\
\hline $\begin{array}{l}\text { Rocha et al } \\
(2010)\end{array}$ & 13 & $\begin{array}{l}\text { Dois meses, três } \\
\text { vezes por semana, } \\
\text { (durante a HD) 25'. }\end{array}$ & $\begin{array}{l}\text { MMSS: técnica FNP, } \\
\text { diagonais de Kabat, } \\
\text { exercício de fortalecimento } \\
\text { com halter, cinesioterapia, } \\
\text { exercício com bola } \\
\text { exercitadora para preensão } \\
\text { manual em MS com fístula. } \\
\text { MMIl: cinesioterapia } \\
\text { resistida com caneleira. } \\
\text { Respiração diafragmática. }\end{array}$ & $\begin{array}{l}\text { Apenas o PFE } \\
\text { mostrou-se } \\
\text { estatisticamente } \\
\text { significante. No } \\
\text { SF-36 observou } \\
\text { melhora dos oito } \\
\text { domínios, exceto do } \\
\text { "vitalidade". }\end{array}$ \\
\hline $\begin{array}{l}\text { Soares et al } \\
(2011)\end{array}$ & 27 & $\begin{array}{l}\text { Três meses, duas } \\
\text { vezes por semana, } \\
\text { (durante a HD) 3O'. }\end{array}$ & $\begin{array}{l}\text { Alongamento e } \\
\text { fortalecimento dos } \\
\text { membros e relaxamento } \\
\text { (conscientização } \\
\text { respiratória). }\end{array}$ & $\begin{array}{l}\text { No SF-36 } \\
\text { mostrou melhora } \\
\text { dos domínios: } \\
\text { capacidade } \\
\text { funcional, nível } \\
\text { de dor, vitalidade } \\
\text { e saúde mental. } \\
\text { Houve relatos de } \\
\text { melhora de cãibras } \\
\text { musculares. }\end{array}$ \\
\hline $\begin{array}{l}\text { Magalhães et } \\
\text { al (2004) }\end{array}$ & 13 & $\begin{array}{l}\text { Quatro meses, duas } \\
\text { vezes por semana, } \\
\text { (durante a HD) 3O'. }\end{array}$ & $\begin{array}{l}\text { Alongamentos de MMSS } \\
\text { e MMIl e fortalecimentos } \\
\text { apenas em MMIl com } \\
\text { caneleiras e diagonais de } \\
\text { Kabat. }\end{array}$ & $\begin{array}{l}\text { da força e } \\
\text { resistência muscular. } \\
\text { cãibras, dores e } \\
\text { parestesias. }\end{array}$ \\
\hline
\end{tabular}


(conclusão)

\begin{tabular}{|c|c|c|c|c|}
\hline AUTORES & AMOSTRA & PERÍODO & EXERCÍCIOS & RESULTADOS \\
\hline $\begin{array}{l}\text { Lopes et al } \\
(2008)\end{array}$ & 15 & $\begin{array}{l}\text { Um mês, três } \\
\text { vezes por semana, } \\
\text { (previamente à HD) } \\
\text { 4O'. }\end{array}$ & $\begin{array}{l}\text { Exercício físico ativo } \\
\text { livre isotônico de baixa } \\
\text { intensidade para MMSS } \\
\text { e MMIl, alongamentos, } \\
\text { diagonais de Kabat, rotação } \\
\text { de tronco com bastão, } \\
\text { agachamento. }\end{array}$ & $\begin{array}{l}\text { Kt/V do mês sem } \\
\text { exercício, com o mês } \\
\text { com exercício não } \\
\text { obteve mudanças } \\
\text { estatisticamente } \\
\text { significantes. } \\
\text { Pacientes relataram } \\
\text { melhora no } \\
\text { comportamento } \\
\text { psicossocial, na } \\
\text { socialização, do } \\
\text { quadro depressivo, } \\
\text { diminuição de } \\
\text { queixas de câimbras, } \\
\text { náuseas e vômitos. }\end{array}$ \\
\hline $\begin{array}{l}\text { Oliveros et al } \\
(2011)\end{array}$ & $\begin{array}{l}15, \text { grupo } \\
\text { controle } \\
(n=6) \text { e } \\
\text { exercício } \\
(n=9)\end{array}$ & $\begin{array}{l}\text { Quatro meses, três } \\
\text { vezes por semana } \\
\text { (durante a HD) 50'. }\end{array}$ & $\begin{array}{l}\text { Aquecimento, exercícios } \\
\text { aeróbicos no ciclo ergômetro } \\
25 \text { ' e de resistência por } 25 \text { ' } \\
\text { como: exercícios isotônicos } \\
\text { em MMIl e de extensão de } \\
\text { joelho com uso de faixa } \\
\text { elástica. }\end{array}$ & $\begin{array}{l}\text { da distância } \\
\text { percorrida no TC6' e } \\
\text { da força muscular de } \\
\text { MMII. }\end{array}$ \\
\hline
\end{tabular}

